\title{
Über die Auflösungsgeschwindigkeit fester Körper.
}

\author{
Von \\ L. Bruner und St. Tommoczko. \\ (Zweite Mitteilung.)
}

I. Experimentelles: über den Einflufs des Volumens und der Drehungsgeschwindigkeit auf die Auflösungsgesehwindigkeit. Anhang: Vorläufige Versuche über den Einflul's der Oberflächenbeschaffenheit des festen Körpers und über die Katalyse bei der Auflösung. II. Theoretisches.

\section{Experimentelles.}

In unserer ersten Mitteilung ${ }^{1}$ haben wir bereits im Anschlul's an die Versuche DE Hews's und Noyes, dafs die Auflösungsgeschwindigkeit proportional der jeweiligen Differenz der Konzentration der Lösung und derjenigen der gesättigten Lösung erfolge, gezeigt und die nach dem hier obwaltenden logarithmischen Gesetz auf $1 \mathrm{ccm}$ sich lösenden Oberfläche bezogenen Konstanten berechnet. Wir haben die Meinung vertreten, dafs es sich hier nur um Diffusionskoëffizienten handele, d. h. dafs unsere Konstanten keine spezifischen Konstanten für die eigentümliche Lösungsreaktion zwischen fester Phase und ungesättigter Lösung sind, sondern lediglich von der Wechselwirkung zwischen der adhärierenden gesättigten Schicht und der ungesättigten Lösung abhängen.

Wir waren bereits mit weiteren Versuchen beschäftigt, als eine Arbeit von Herrn DRUCKER ${ }^{2}$ erschien, die uns veranlafst hat, die weiter unten angegebenen Versuche auszugsweise mitzuteilen, um einige von Herrn Drucker erhobene Einwände, mit denen wir uns nicht im ganzen einverstanden erklären können, zu entkräften. Herr Drucker ist ja der Ansicht, dafs die Auflösungsgeschwindigkeit vom

1 Zeitschr. phys. Chem. 35, 281.

Zeitscher. phys. Chen. 36, 693. 
Volumen abhängig und zwar demselben umgekehrt proportional ist Wir werden später auf die theoretische Ableitung dieses seines Schlusses eingehen, nachdem wir die Resultate unserer darauf gerichteten Versuche mitgeteilt haben werden, da wir glauben, dafs in einem so wenig erforschten Falle dem Experiment das erste Wort gebührt. Wie wir sehen werden, wird der Schlufs keineswegs vom Experiment bestätigt und die Abhängigkeit vom Volumen ist nur eine scheinbare; die widersprechenden Druckek'schen Versuche werden sich wohl anders erklären lassen. Dagegen hat Herr Drucker ganz mit Recht darauf hingewiesen, dafs die Auflösungsgeschwindigkeit von der Rührgeschwindigkeit des Wassers abhängig sein muls, indem dadurch die Beschaffenheit der adhärierenden Schicht modifiziert sein kann. Der Rührvorrichtung ist daher nicht nur die Rolle einer Vorrichtung zum relativ momentanen Konzentrationsausgleich im Inneren der Lösung zuzuschreiben, sondern dieselbe übt einen wesentlichen Einflufs auf die Auflösungsgeschwindigkeit, dem durch Versuche nachzuspüren ist.

Die von uns ausgeführten Versuche bezwecken folglich die Abhängigkeit der Auflösungsgeschwindigkeit 1. vom Gesamtvolumen der Lösung, 2. von der Rührgeschwindigkeit (Form des Gefälses, Form der Rührvorrichtung mit inbegriffen) zu erforschen.

Versuche mit Benzoësäure. Wir haben zuerst einige Versuche mit Benzoësäure angestellt, die wir, wie in der ersten Mitteilung angegeben, in Platten gegossen haben. Die Platten sind recht hart und gegen Bruch widerstandsfähig und können leicht bis $30 \mathrm{qcm}$ Oberfläche erhalten werden. Es ist sonderbar, dals die Herstellung solcher Platten Herrn DRuoksR milslang. Wir behielten die frühere Versuchsanordnung bei, wie sie in unseren ersten Versuchen zur Anwendung kam. Das Gefäls, in dem die Auflösung erfolgte, war ein dickwandiges cylindrisches Becherglas von $10.5 \mathrm{~cm}$ Durchmesser. Ein Liter Wasser hatte darin die Höhe von $12.5 \mathrm{~cm}$ (Gefäls A). Auf die Einhaltung der konstanten Temperatur von $24.8^{\circ}$ im Thermostaten (Normalthermometer) war grofse Sorgfalt verwendet. Schon in dem ersten Versuche haben wir uns überzeugt, dal's die Konstante pro $1 \mathrm{ccm}$ viel grölser ausfällt, als wir sie früher gefunden haben, und einen regelmälsigen aufsteigenden Gang aufweist. Es sind nämlich statt des früheren Wertes $D=0.00440$ Zahlen von 0.0062 bis 0.0100 gefunden worden. Wir machten zugleich die Beobachtung, dafs die Titrierung mit $\mathrm{Ba}(\mathrm{OH})_{2}$ nicht mit der gewöhnlichen Schärfe erfolgte, sondern nach eingetretener Rotfärbung in kurzer Zeit die 
Farbe wieder verschwand. Wir kamen daher auf den Gedanken, dafs trotz der scheinbar vollkommenen Klarheit der Lösung die geschwinde Drehung (bis $600 \mathrm{mal}$ pro Minute) die Abspaltung kleiner Teilchen festen Stoffes bewirke, wodurch der Titer erhöht wird, ohne dafs dies auf grölsere Lösungsgeschwindigkeit zu deuten wäre. In der That haben wir uns überzengt, dafs diese Vermutung richtig war. Bei einer mälsigen Rührgeschwindigkeit von ca. $200 \mathrm{mal}$ pro IMinute nach 90 Minuten andauerndem Rührens stellte sich z. B. der Titer einer entnommenen Probe von $20 \mathrm{ccm} \mathrm{zu}$ : unfiltriert $4.65 \mathrm{ccm}$, filtriert $3.70 \mathrm{ccm} \mathrm{Ba}(\mathrm{OH})_{2}{ }^{1}$ Bei kräftigerem Rühren (bis 600 pro Minute) nach Verlauf von zwei Stunden wurde z. B. der Titer gefunden: filtriert $3.73 \mathrm{ccm}$, unfiltriert $9.12 \mathrm{ccm} \mathrm{Ba}(\mathrm{OH})_{2}$. Bei grofser Rührgeschwindigkeit machen die mikroskopisch kleinen, unsichtbaren suspendierten Teilchen bei weitem den grölsten Teil der alkalimetrisch bestimmten "Löslichkeit" aus. Dies wird natürlich um so mehr der Fall sein, je weniger fest die Platten hergestellt sind; nun giebt von den seinigen Herr Drucker besonders an, dals sie eine körnige Struktur besalsen und, falls sie dünner als $1 \mathrm{~cm}$ gewesen sind, beim Rühren zerfielen. Die unserigen waren etwa 0.2-0.3 cm dick und einen Zerfall oder makroskopische Suspension konnten wir nicht beobachten; trotzdem ist eine solche sicher vorhanden. Dals dies bei den Versuchen Drucker's der Fall sein könnte, darf als recht wahrscheinlich angesehen werden. Bei der Ausführung unserer Versuche haben wir deshalb jede entnommene Probe direkt durch einen trockenen Filter filtriert und der durch Adhäsion der Lösung an dem Filter bewirkten Verkleinerung des Titers dadurch Rechnung getragen, dal's wir für $C$ ebenso den Titer einer gesättigten und durch trockenen Filter filtrierten Lösung gesetzt haben. Der Unterschied ist jedenfalls recht gering, ca. $1-2 \%$. Denn es verbrauchen $20 \mathrm{~cm}$ einer gesättigten Lösung 12.60, 12.64, im Mittel $12.62 \mathrm{~cm} \mathrm{Ba}(\mathrm{OH})_{2}\left(1 \mathrm{ccm} \mathrm{Ba}(\mathrm{OH})_{2}\right.$ entspricht 0.04384 Millimol der Säure); ${ }^{2}$ filtriert dagegen nur 12.28, 12.26, 12.15, im Mittel $12.23 \mathrm{ccm} \mathrm{Ba}(\mathrm{OH})_{2}$.

Wir lassen jetzt die Versuchsergebnisse folgen. In den Tabellen ist die frühere Bezeichnung beibehalten, und es bedeuten:

1 Titer des $\mathrm{Ba}(\mathrm{OH})_{2}: 1 \mathrm{ccm}$ entspricht 0.04384 Millimol Benzoësäure.

2 Es ergiebt sich daraus die Löslichkeit der Benzoësäure bei $24.8^{\circ} \mathrm{zu}$ 36.15 Liter pro $1 \mathrm{Mol}$ Säure; in Übereinstimmung mit Drucker, der bei $25^{\circ}$ 36.52 Liter findet. 
$f$ die Gesamtoberfläche der Platten,

$C$ die Konzentration der gesättigten Lösung (filtriert),

$x$ die Konzentration der Lösung für die Zeit $t$,

$t$ die Zeit in Stunden,

$D$ die Geschwindigkeitskonstante pro $1 \mathrm{qcm}$ Oberfläche, berechnet nach der Formel:

$$
D=\frac{1}{f t} \log \frac{C}{C-x}, \text { es ist also } D=0.4343 D_{n},
$$

wo $D_{n}$ die Konstante bedeutet, die sich durch Integration der Differentialgleichung $d x / d t=D_{n}(C-x)$ ergeben mülste. Da bei unserer Untersuchung es sich nur um relative mit einander zu vergleichende Zahlen handelt, haben wir, wie üblich, mit den dekadischen Logarithmen gerechnet.

Den Tabellen sind Angaben über das Anfangsvolum ( $V)$ und die mittlere Drehgeschwindigkeit der Rührvorrichtung beigefügt. Wegen der kleinen Arbeitsfähigkeit unseres Motors (HEINRICI'scher Luftmotor Nr. 1, 1/80 HP) dieselbe genau zu bestimmen und sicher konstant zu erhalten, erscheint als recht schwierig. Während eines jeden Versuches haben wir die Grölse der heizenden Flamme des Brenners unverändert gelassen und in regelmälsigen Zeitintervallen die Maschine mit Petroleum geschmiert. Die Bestimmung der Tourenzahl, da der Tourenzähler den Motor stark gehemmt hatte, geschah, indem wir die Umlaufszeit der gleitenden Schnur beobachtet und aus dem Verhältnis ihrer Länge zum Durchmesser der Axe die Umdrehungszahl pro Minute berechnet haben. Wir sind uns wohl bewufst, dafs diesen Bestimmungen ziemlich viel Unsicherheit anhaftet und werden uns bemühen, wenn uns stärkere Kraftquellen zur Verfügung stehen werden, dieselben einwurfsfrei und streng angeben zu können.

\section{Tabelle 1.}

Volum $=1000 \mathrm{ccm}$ (Gefäls $\mathrm{A}) C=12.23 \mathrm{ccm} \mathrm{Ba}(\mathrm{OH})_{2}$ pro $20 \mathrm{~cm}$ gesätt. Lösung $f=13.72 \mathrm{qcm} \quad C_{1}-x_{1}=9.26 \mathrm{ccm} \mathrm{Ba}(\mathrm{OH})_{2}{ }^{1}$ Tourenzahl ca. 450 pro Min.

$\begin{array}{lllc}t & x & C-x & D \\ 1 & 4.45 & 7.78 & 0.00552 \\ 1.50 & 5.02 & 7.21 & 0.00530 \\ 2.50 & 6.15 & 6.08 & 0.00535 \\ 3.50 & 7.04 & 5.19 & 0.00524\end{array}$

${ }^{1}$ Da im Anfange des Versuches eine der Platten von dem Flügel des Rührers abgefallen war, mufste der Versuch unterbrochen werden und ist weiter wie üblich nach der Formel $D=\frac{1}{f t} \log \frac{C-x}{C-x}$, wenn zur Zeit $t_{0}=0, x_{1}=2.97 \mathrm{cem}$ gehört, berechnet worden. 
Tabelle 2.

Volum $=1000 \mathrm{ccm}($ Gefäls A $) \quad O=12.23 \mathrm{~cm} \mathrm{Ba}(\mathrm{OH})_{2}$ pro $20 \mathrm{ccm}$ ges. ᄃösung $f=14.97$ qcm Tourenzahl ca. 450 pro Minute

\begin{tabular}{cccc}
$t$ & $x$ & $C-x$ & $D$ \\
0.50 & 1.10 & 11.13 & 0.00547 \\
1.00 & 2.02 & 10.21 & 0.00523 \\
1.50 & 2.88 & 9.35 & 0.00519 \\
2.00 & 3.73 & 8.50 & 0.00527 \\
3.00 & 5.15 & 7.08 & 0.00527 \\
4.00 & 6.45 & 5.78 & 0.00543 \\
\cline { 3 - 4 } & & Mittel aus Tabelle 1, 2: 0.005333
\end{tabular}

Als Auflösungskonstante erhalten wir im Mittel 0.00533, während unsere früheren Versuche 0.00440 ergeben haben. Der nene Wert steht dem früheren wohl nicht allza fern und die bestehende Differenz, die jedoch die Versuchsfehler übersteigt, liefse sich zurückführen: 1. auf die grölsere Drehgeschwindigkeit und 2. auf die Vergröfserung der sich lösenden Oberfläche, da die suspendierten Teilchen mit ihren unberechenbaren Flächen wohl auch in dem Auflösungsvorgange mitspielen. Dies ist um so mehr wahrscheinlich, als unsere früberen Versuche, ob sie unfiltriert oder zu Kontrollzwecken filtriert gewesen sind, übereinstimmende Titer ergaben. Jedenfalls ist der Wert 0.00533 fast dreimal kleiner als derjenige Drucker's. Die Übereinstimmung, die Herr Drucker mit unserem früheren Wert $0.00440 \mathrm{zu}$ finden geglaubt hat, beruht nur auf einen Rechenfehler, indem er annahm, dafs wir mit log. den natürlichen Logarithmus bezeichnet haben und zugleich die Zeit in Minnten (nicht wie wir in Stunden) rechnete, dafür aber eine Dezimalstelle bei seiner Rechnung durch Versehen wegliefs. Rechnet man seine Versuche um, so erhält man als Konstante pro 1 Liter Lösung 0.0122, während wir übereinstimmend aus zwei Reihen 0.00533 finden. Wir glauben diesen Unterschied dadurch erklären zu dürfen, dals Herr Drucker auf die Suspension fester Teilchen nicht die notwendige Rücksicht genommen hat.

Um den Einflufs der Volumänderung zu untersuchen, haben wir einen Versuch in der Weise angestellt, dafs wir nach zwei bis drei entnommenen Proben einen beträchtlichen Teil der Lösung herauspipettiert haben: ${ }^{1}$ bei weiter erfolgender Auflösung sollte man nach Druсkek ein Steigen der Konstante $D$ erwarten.

1 Diese Art des Versuches haben wir vorgezogen, um den Schwankungen der Drehgesehwintigkeit mäglichst vorzubeugen. Bei der angegebenen Anord- 
Tabelle 3.

\begin{tabular}{|c|c|c|c|}
\hline \multicolumn{2}{|c|}{ Volum $=1000 \mathrm{ecm}($ Gefäls A $)$} & $f=\begin{aligned} 18.91 \mathrm{qcm} \\
\text { Tourenzah }\end{aligned}$ & $\begin{array}{l}C=12.23 \mathrm{~cm} \mathrm{Ba}(O H)_{2} \\
\text { ca. } 450 \text { pro Minute }\end{array}$ \\
\hline$t$ & $x$ & $C-x$ & $D$ \\
\hline 0.50 & 1.33 & 10.90 & 0.00527 \\
\hline 1.00 & 2.45 & 9.78 & 0.00512 \\
\hline
\end{tabular}

nach $t=1.00$ sind $300 \mathrm{cem}$ herauspipettiert worden $V=500 \mathrm{ccm}$

$\begin{array}{llrl}1.50 & 3.64 & 8.59 & 0.00538 \\ 2.00 & 4.70 & 7.53 & 0.00553\end{array}$

nach $t=2.00$ sind noch $100 \mathrm{ccm}$ herauspipettiert worden $V=650 \mathrm{ccm}$

$\begin{array}{llll}3.25 & 6.61 & 5.62 & 0.00545 \\ 4.00 & 7.52 & 4.71 & 0.00545\end{array}$

Dies wird von dem Versuch keineswegs bestätigt: die Konstante hat keinen aufsteigenden Gang. Der Versuch stimmt mit unseren früheren überein, in denen wir auch nach Entnahme von $40 \%$ des ganzen Volumens kein Steigen der Konstante gefunden baben. Woher kommt denn das abweichende Resultat der Drucker'schen Versuche zu stande? Nehmen wir einmal an, dafs die von ihm bestimmten Titer vorzugsweise durch Suspension fester Teilchen verursacht gewesen sind, so ist leicht ersichtlich - da die Menge abgespalteter Teilchen nur durch die Drehgeschwindigkeit und die Beschaffenheit der Platten gegeben wird, dafs der Titer in einem kleinen Volumen und daher auch die „Auflösungsgeschwindigkeitskonstante" grölser als in einem grofsen Volumen der Lösung erscheinen müssen. Ess wäre sehr interessant zu erfahren, ob Herr Drucker seine Proben vor der Analyse filtriert und überhaupt der Homogenität der Lösung die nötige Rechnung getragen hat.

Über den Einflufs der Drehgeschwindigkeit giebt uns die Tabelle 4 Auskunft.

Tabelle 4.

\begin{tabular}{cccc}
$\begin{array}{c}\text { Volum }=1000 \mathrm{~cm} \\
(\text { Gefäls A) }\end{array}$ & \multicolumn{2}{c}{$f=18.91 \mathrm{qcm}$} & \multicolumn{2}{c}{$C=12.23 \mathrm{~cm} \mathrm{Ba}(\mathrm{OH})_{2}$} \\
Tourenzahl ca. & 100 pro Minute \\
$t$ & $x$ & $C-x$ & $D$ \\
0.50 & 0.75 & 11.48 & 0.00290 \\
1.00 & 1.43 & 10.80 & 0.00287 \\
1.50 & 2.09 & 10.14 & 0.00288 \\
3.50 & 4.18 & 8.05 & 0.00273 \\
5.00 & 5.48 & 6.75 & 0.00273 \\
6.50 & 6.61 & 5.62 & 0.00265 \\
& & & Mittel: 0.00279
\end{tabular}

nung erhalten wir aus einem und demselben Versuche die Konstante für zwei verschiedene (1 Liter, $500 \mathrm{cem}$ ) Volumina. 
Die Konstante ist jetzt auf den halben Wert gesunken; auch ist, wie wir uns durch Kontrollproben überzeugt haben, der Unterschied zwischen filtrierten und unfiltrierten Proben weit geringer als in den früheren Versuchen. Dals die Drehgeschwindigkeit in Tabelle 4 zur Konvektion im Inneren der Lösung ausreichend war, ist auch kontrolliert worden.

Wir haben keine weiteren Versuche mit Benzoësäure angestellt, da wir beabsichtigten, ferner mit möglichst rascher Drehgeschwindigkeit zu experimentieren, um den unvermeidlichen Schwankungen der Konstante vorzubeugen, und bei solchen Versuchen ist die Benzoësäure wenn auch brauchbar, so doch ziemlich unbequem, da eine genaue Bestimmung der Oberfläche nicht durchzuführen ist.

Versuche mit Gips. Wir haben deshalb weitere Versuche mit einem anderen Stoffe, nämlich mit Gips, ausgeführt. Statt der Gipskrystalle haben wir, wo nicht besonders angegeben, reinsten Alabaster verwendet, aus dem sich Platten von jeder gewünschten Grölse ganz einfach mittels der Laubsäge schneiden lassen. Die Platten sind mit feinem Glaspapier und endlich auf mattem Glase abgeschliffen worden. Auch bei lange dauernden Versuchen ist die Erosion solcher Platten nur eine geringe gewesen. Die Analyse der Proben geschah durch Abdampfen, mälsiges Glühen des Rückstandes und Wägen des zurückgebliebenen $\mathrm{CaSO}_{4}$.

Wir haben zuerst einige Versuche in demselben Gefälse (A) angestellt, die Resultate sind in den Tab. 5 und 6 zusammengestellt.

\section{Tabelle 5 .}

Volum $=1000 \mathrm{cem} f=21.40 \mathrm{qcm} \quad C=0.0530 \mathrm{~g} \mathrm{CaSO}_{4} .2 \mathrm{H}_{2} \mathrm{O}$ pro $20 \mathrm{~cm}$ ges. Lösung

(Gefäls A)

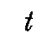

0.50

1.00

1.50

2.00

3.50

4.50

5.50

7.00 $x$

0.0051

0.0100

0.0141

0.0190

0.0289

0.0333

0.0365

0.0406
Tourenzahl ea. 450 pro Minute

$$
C-x \quad D
$$

$\mathbf{0 . 0 4 7 9} \quad \mathbf{0 . 0 0 4 1 0}$

$0.0430 \quad 0.00424$

$0.0389 \quad 0.00418$

$0.0340 \quad 0.00451$

$0.0241 \quad 0.00458$

$0.0197 \quad 0.00449$

$0.0165 \quad 0.00431$

$0.0124 \quad 0.00421$ 
Tabelle 6 .

Volum $=100 \mathrm{ccm} \quad f=18.92 \mathrm{qcm} \quad C=0.0419 \mathrm{~g} \mathrm{CaSO}$ pro $20 \mathrm{ccm}$ ges. Lösung (Gefäls A)

$\begin{array}{ll}t & x \\ 1.50 & 0.0118 \\ 2.50 & 0.0160 \\ 3.50 & 0.0217 \\ 4.50 & 0.0258 \\ 5.50 & 0.0292 \\ 6.50 & 0.0319 \\ 7.50 & 0.0329\end{array}$

Tourenzahl ca. 450 pro Minute

\begin{tabular}{cc}
$C-x$ & $D$ \\
0.0301 & 0.00506 \\
0.0259 & 0.00442 \\
0.0202 & 0.00478 \\
0.0161 & 0.00488 \\
0.0127 & 0.00500 \\
0.0100 & 0.00506 \\
0.0090 & 0.00468 \\
\hline
\end{tabular}

Vom Alabaster werden keine festen Teilchen abgespalten, da die Proben, filtriert oder unfiltriert, identische Wägungen ergaben. Die Schwankungen der Konstanten sind ziemlich grofs; der Versuch in der Tabelle 6 zeigt durchschnittlich höhere. Werte als der Versuch in der Tabelle 5. Dies mag auf den Drehverhältnissen beruhen; andererseits sind die zu wägenden Quantitäten sehr klein, wodurch die Versuchsfehler ganz bedeutend gesteigert werden können.

Trotzdem dafs in dem Versuche (Tabelle 6) das Volumen der Lösung durch Herauspipettieren von Kontrollproben auf $700 \mathrm{ccm}$ gesunken ist, ist kein bestimmtes Anzeigen für ein Steigen der Konstante vorhanden. Die Konstante ist beträchtlich grölser als die in unserer ersten Mitteilung angegebene. Diese Verschiedenheit beruht auf der Verschiedenheit des Versuchsmateriales, da wir früher Gipskrystalle (Marienglas) angewendet haben; wie wir später sehen werden, ist die Auflösungsgeschwindigkeit des Marienglases etwa dreimal kleiner als diejenige des Alabasters.

In der Tabelle 7 ist das Resultat eines Versuches angegeben, der ebenso wie bei der Benzoësäure (Tabelle 3) über den Einflufs des Volumens angestelit worden ist.

\section{Tabelle 7.}

Volum $=1000 \mathrm{~cm} f=22.16 \mathrm{qcm} \quad C=0.0530 \mathrm{~g} \mathrm{CaSO}_{4} .2 \mathrm{H}_{2} \mathrm{O}$ pro $20 \mathrm{cem}$ ges. Lösung (Gefäl's A)

Tourenzahl ca. 450 pro Minute

\begin{tabular}{|c|c|c|c|}
\hline$t$ & $x$ & $C-x$ & $D$ \\
\hline 1.50 & 0.0150 & 0.0380 & 0.00435 \\
\hline \multicolumn{4}{|c|}{ nach $t=1.50$ sind $300 \mathrm{ecm}$} \\
\hline 2.50 & 0.0233 & 0.0297 & 0.00450 \\
\hline 3.50 & 0.0289 & 0.0241 & 0.00441 \\
\hline 4.50 & 0.0333 & 0.0197 & 0.00430 \\
\hline
\end{tabular}

nach $t=4.50$ sind noch $75 \mathrm{cem}$ herauspipettiert worden.
7.00
0.0411
0.0119
0.00418

Z. anorg. Chem. XXVIII. 
Auch hier ist kein Anzeigen für das Wachsen der Konstante.

Dals die Umdrehungsgeschwindigkeit einen grofsen Einflufs auf die Auflösungsgeschwindigkeit übt, ist schon aus den Versuchen mit Benzoësäure (Tabelle 4), ebenso wie aus der Tabelle $8 \mathrm{zu}$ entnehmen.

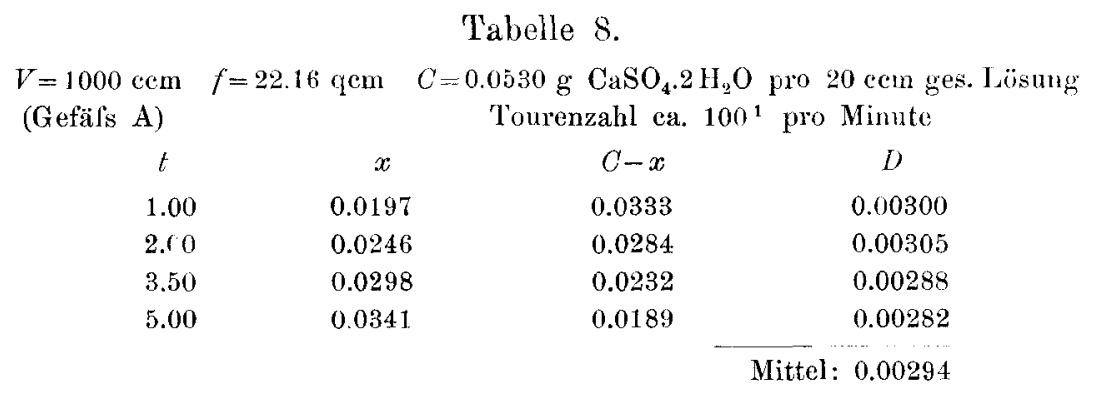

Um einen entscheidenden Beweis über den Volumeinflul's zn erbringen, haben wir weitere Versuche in einem anderen grölseren Gefälse angestellt. Das Gefäls B (ein grofses Becherglas) hatte den Durchmesser von $14 \mathrm{~cm}$ und die Wassermenge von 3 Liter hatte darin die Höhe von $22 \mathrm{~cm}$. Zur Analyse haben wir zu gleicher Zeit 2 Proben zu $50 \mathrm{ccm}$ entnommen, von denen zu Kontrollzwecken, falls Unsicherheit vorlag, jede abgedampft und gewogen wurde.

In den Tabellen 9 und 10 sind die Resultate zweier Versuche wiedergegeben, die, wie wir glauben, entschieden gegen die DRUCKen'sche Auffassung sprechen. Die Anfangswerte, die wir einmal mit 2 und dann mit 3 Liter Wasser erhalten haben, zeigen eine vollkornmene Übereinstimmung, wo wir im zweiten Falle nach Herrn Drdcker eine Abnahme der Konstante um 50\% zu erwarten hätten. Dieses Resultat auf die Verschiedenheit der Drehgeschwindigkeit beziehen zu wollen, ist unstatthaft, denn da wir in beiden Reihen die ganze Leistungsfähigkeit unseres Motors bethätigt haben, die Konstante für 3 Liter eher zu klein als zu grols ausfallen könnte.

1 Wegen einer zufälligen Unterhrechung des Versuches muliste dieser vom Anfangswert $t_{0}=0, x_{0}=0.0143, C-x_{0}=0.0387$ nach der Formel:

$$
D={ }^{1} / f \cdot t \cdot \log \cdot \frac{C-x_{0}}{U-x}
$$

berechnet worden sein. 
Tabelle 9.

$V=2000 \mathrm{cem} \quad f=22.91 \mathrm{qcm}$ (Gefäl's B)

$\begin{array}{cc}t & x \\ 1.50 & 0.0173 \\ 3.00 & 0.0322 \\ 4.00 & 0.0392 \\ 5.00 & 0.0475 \\ 6.00 & 0.0543 \\ 7.00 & 0.0602 \\ 8.00 & 0.0662\end{array}$

$C=0.1047 \mathrm{~g} \mathrm{CaSO}_{4}$ pro 50 cern ges. Lüsung Tourenzahl ca. 400 pro Minute

$\begin{array}{cc}C-x & D \\ 0.0874 & 0.00229 \\ 0.0725 & 0.00231 \\ 0.0655 & 0.00222 \\ 0.0572 & 0.00229 \\ 0.0504 & 0.00230 \\ 0.0445 & 0.00231 \\ 0.0385 & 0.00236 \\ & - \text { Mittel }: 0.00230\end{array}$

Tabelle 10.

\begin{tabular}{|c|c|c|c|}
\hline$V=3000 \mathrm{ccm}$ & $f=22.91 \mathrm{qcm}$ & \multicolumn{2}{|c|}{$\begin{array}{c}C=0.1047 \mathrm{~g} \mathrm{CaSO}_{4} \text { pro } 50 \mathrm{ccm} \text { ge } \\
\text { Tourenzahl ca. } 400\end{array}$} \\
\hline$t$ & $x$ & $C-x$ & $D$ \\
\hline 1.50 & 0.0174 & 0.0873 & 0.00229 \\
\hline 2.50 & 0.0272 & 0.0775 & 0.00227 \\
\hline 3.50 & 0.0347 & 0.0700 & 0.00219 \\
\hline
\end{tabular}

nach $t=3.50$ sind $450 \mathrm{cem}$ herauspipettiert worden.

$V=2250 \mathrm{~cm}$

$\begin{array}{llll}5.00 & 0.0452 & 0.0595 & 0.00214 \\ 6.00 & 0.0525 & 0.0522 & 0.00220 \\ 7.00 & 0.0572 & 0.0475 & 0.00214\end{array}$

nach $t=7.00$ sind $300 \mathrm{cem}$ herauspipettiert worden.

$V=1600 \mathrm{ccm}$

$\begin{array}{rlll}9.00 & 0.0689 & 0.0358 & 0.00226 \\ 10.00 & 0.0754 & 0.0293 & 0.00241 \\ 11.00 & 0.0788 & 0.0259 & 0.00240\end{array}$

Dafs die Konstanten in den Tabellen 10 und 11 etwa den halben Wert von denen für 1 Liter Lösung erhaltenen erreichen, ist ganz selbstverständlich durch die verminderte Drehgeschwindigkeit einer so grofsen Menge Wasser in einem viel breiteren Gefälse verursacht. Da, wie oben angegeben, die Drehgeschwindigkeit von entscheidendem Einflusse auf die Auflösungsgeschwindigkeit ist, eine konstante Drehgeschwindigkeit aber mit dem kleinen Luftmotor zu erzielen nicht durchführbar ist, so haben wir uns mit den Versuchen in der Tabelle 9 und 10 begnügt, da der Einwand immer gemacht werden könnte, dals die Resultate durch den zahlenmälsig nicht kontrollierbaren Einflufs der Rührgeschwindigkeit entstellt worden sind. Wir glauben jedoch, dafs in diesen Versuchen (Tabelle 10 und 11) die 
Unabhängigkeit der Lösungsgeschwindigkeit vom Volumen genügend dargethan ist. Wir trachteten die Unabhängigkeit der Auflösungsgeschwindigkeit vom Volumen noch einmal zu verifizieren, nachdem wir durch passende viel schnellere Drehungen konstante Auflösungsgeschwindigkeit erzwingen wollten. Wir setzten nämlich voraus, dals die Auflösungsgeschwindigkeit bei steigender Drehgeschwindigkeit der Wassermenge sich einem wohl erreichbaren Maximum nähern würde. Mit dem kleinen Luftmotor, der uns zur Verfügung stand, konnten wir nicht schnellere Drehungen, als oben angegeben, erzielen. Wir haben daher weitere Versuche mit einer RABE'schen Turbine angestellt. Nit dieser Turbine konnten wir die Umdrehgeschwindigkeit bis $600-700$ pro Minute steigern; auch in dem Falle ist es schwer, sie konstant zu erhalten, denn jede Benutzung der Wasserleitung in den Arbeitsräumen wie auf der Stralse verringerte den Wasserzuflufs und folglich auch die Drehgeschwindigkeit, besonders wenn von der Turbine, wie in unserem Falle, grofse Arbeitsleistung verlangt wurde. Der Wirbel, der sich bei so raschen Umdrehungen des Rührers bildete, war so tief, dafs die Platten nicht mehr total mit Wasser bedeckt wurden; wir haben deshalb die Platten an einem aus Messing gefertigten Ringe angebracht; der Ring hatte den Durchmesser des Becherglases und war an diesem so befestigt, dafs die Platten in die halbe Höhe der Wassersäule kamen. Die Flügel des Rührers sind durch Anbringen von Messingplatten vergröfsert worden. Die Tabelle 11 giebt uns über diesen. Versuch Auskunft.

Tabelle 11.

$\begin{array}{cccc}V=2250 & f=21.60 \mathrm{qcm} & \begin{array}{c}C=0.1047 \mathrm{~g} \mathrm{CaSO} \\ \text { Tourenzahl ca. }\end{array} \text { pro } 50 \mathrm{ccm} \text { ges. Lösung } \\ (\text { Gefäls B) } & & C-x & D \\ t & x & 0.0612 & 0.00420 \\ 2.00 & 0.0435 & 0.0525 & 0.00431 \\ 2.50 & 0.0522 & 0.0450 & 0.00436 \\ 3.00 & 0.0597 & 0.0385 & 0.00441 \\ 3.50 & 0.0662 & 0.0332 & 0.00443 \\ 4.00 & 0.0715 & 0.0272 & 0.00432 \\ 4.50 & 0.0775 & & \end{array}$

Mittel: 0.00434

Die Konstante ist auf den doppelten Wert, den sie bei den Versuchen mit 2 und 3 Liter hatte, gestiegen. Der Wert nähert sich demjenigen, den wir bei den früheren Versuchen mit 1 Liter 
Lösung (Gefüls A) gefunden haben. Auf einige vorläufige Versuche, wo wir Werte für $D=$ bis 0.006 gefunden haben, gestützt, glauben wir jedoch, dafs wir das Maximum der Auflösungsgeschwindigkeit des Alabasters noch nicht erreicht haben. Kleinere Drehgeschwindigkeiten zu untersuchen bietet für uns nur ein geringes Interesse so lange, als die uns zu Gebote stehenden Mittel dieselbe absolut konstant $\mathrm{zu}$ erhalten und genügend weit zu treiben nicht gestatten. Für die erforderlichen sehr raschen Drehungen glauben wir als passendste Anordnung die in der Tabelle 11 angegebene zu wählen mit dem Unterschiede, dafs wir das Wasser samt dem Becherglase kräftig an einer Axe rotieren lassen, die Platten dagegen unbeweglich erhalten werden. Sobald die nötige Vorrichtung vollendet wird, hoffen wir baldigst weiteres mitteilen zu können, und möchten gern das Thema für gewisse Zeit uns überlassen sehen.

Anhang. Einflufs der Gipsart. Etwaige katalytische Einflüsse. Über die genannten Fragen haben wir einige vorläufige Versuche angestellt, die nicht ausführlicher angelegt worden sind, da wir hoffen, nochmals dieselben mit der maximalen, d. h. mit der von der Rührgeschwindigkeit nunmehr unabhängigen Auflösungsgeschwindigkeit durchzuführen.

Versuch mit Marienglas.

Tabelle 12.

\begin{tabular}{|c|c|c|c|}
\hline $\begin{array}{c}V=2400 \mathrm{ccm} \\
(\text { Gefäl's B) }\end{array}$ & $f=20.90 \mathrm{qem}$ & $\begin{array}{r}C=0.1047 \mathrm{~g} \mathrm{C} \\
\text { Touren }\end{array}$ & $\begin{array}{l}0 \mathrm{ccm} \text { ges. Lösung } \\
\text { ro Minute }\end{array}$ \\
\hline$t$ & $x$ & $C-x$ & $D$ \\
\hline 3.00 & 0.0121 & 0.0926 & 0.00085 \\
\hline 5.00 & 0.0179 & 0.0868 & 0.00078 \\
\hline 6.00 & 0.0210 & 0.0837 & 0.00078 \\
\hline 7.50 & 0.0259 & 0.0788 & 0.00079 \\
\hline \multirow[t]{2}{*}{9.00} & 0.0294 & 0.0753 & 0.00076 \\
\hline & & & 0.00079 \\
\hline
\end{tabular}

Dieser Versuch ist selbstverständlich mit den Versuchen in den Tabellen 10 und 11 vergleichbar, und beweist, dafs die Auflösungsgeschwindigkeit des Marienglases etwa dreimal kleiner ist als die des Alabasters. Dies stimmt mit dem in unserer ersten Mitteilung gewonnenen Resultate überein, das seinerseits mit den Versuchen in den Tabellen 5 und 6 vergleichbar ist. 
Übt die Beschaffenheit der sich lösenden Obertläche einen Einflu's auf die Löslichkeit aus, wie es OsTwnLD ${ }^{1}$ und HuLxTT ${ }^{2}$ nachgewiesen haben, so ist ein solcher anch auf die Auflösungsgeschwindigkeit bei der angewandten Anordnung bemerkt worden. Seine Diskussion wird im zweiten T'eile gegeben.

Katalytische Einflüsse. Für die Auflösungsgeschwindigkeit des Arsentrioxyds - die übrigens wenig Analogie mit dem Auflösungsvorgange des Gipses und anderer von uns untersuchten Stofie hat, da sie ganz ausnahmsweise der logarithmischen Formel nicht gehorcht, was Herr Drucker unserer Meinung nach nicht genügend gewürdigt hat - hat dieser Forscher zahlreiche Katalysenfälle gefunden. Da wir in unserem Falle, wie überhaupt in jeden einfachen Lösungsvorgange das Vorkommen von Katalyse für sehr unwahrscheinlich halten, haben wir uns begnügt, nur einen Versuch über die H-Ionen anzustellen und haben dazu, um jeder chemischen Wechselwirkung vorzubeugen, Schwefelsäure als Katalysator verwendet.

Tabelle 13.

\begin{tabular}{|c|c|c|c|}
\hline $\begin{array}{c}V=2400 \mathrm{cem} \\
(G \text { Gë̈rs B })\end{array}$ & $f=21.32 \mathrm{qcm}$ & \multicolumn{2}{|c|}{$\begin{array}{c}C=0.1047 \mathrm{~g} \mathrm{CaSO}_{4} \text { pro } 50 \mathrm{ecm} \text { ges. } \mathrm{Lüsung} \\
\text { Tourenzahl } 400 \text { pro Minute } \\
\text { Titer der } \mathrm{H}_{2} \mathrm{SO}_{4}=0.04 \text { normal }\end{array}$} \\
\hline$t$ & $x$ & $C-x$ & $D$ \\
\hline 2.00 & 0.0216 & 0.0831 & 0.00235 \\
\hline 3.00 & 0.0319 & 0.0728 & 0.00246 \\
\hline 4.00 & 0.0393 & 0.0654 & 0.00238 \\
\hline 5.00 & 0.0472 & $0.057 \tilde{5}$ & 0.00243 \\
\hline & & & 0.00240 \\
\hline
\end{tabular}

Die Zahlen stimmen mit den bei Abwesenheit des Katalysator's gewonnenen in der Fehlergrenze überein. Man darf daher wohl schliefsen, dals die H-Ionen keine Katalyse hier bewirken. Der sehr kleine Unterschied der Konstanten ist sicher dadurch verursacht, dafs sich von der metallischen Axe, trotzdem sie mit Karadabalsam sorgfältig überzogen wurde, Spuren des Metalls in der Schwefelsäure gelöst haben. Der ausgeglühte $\mathrm{CaSO}_{4}$ zeigte einen Stich ins Rötliche, weshalb der Versuch (Tabelle 13) bei der vierten Probe unterbrochen wurde.

1 Zeitschr. phys. Chem. 34, 495.

${ }^{2}$ Zeitsehr. phys. Chem. 37, 385. 
Mit OH-Ionen haben wir keine Versuche angestellt, da solche wegen der Schwerlöslichkeit von $\mathrm{CaCO}_{3}$ besonderer Vorsichtsmafsregelı bedürfen, um die Lösung vor $\mathrm{CO}_{2}$-Zutritt zu schützen.

Dafs weder $\mathrm{Ca}$ noch $\mathrm{SO}_{4}$-Ionen einen katalytischen Einflufs auf die Lösungsgeschwindigkeit ausüben können, ist schon aus der logarithmischen Form der Gleichung, die wir überall bestätigt gefunden haben, ersichtlich.

\section{Theoretisches.}

Ein prinzipieller Unterschied zwischen unserer Auffussung der Konstanten, die für die Auflösungsgeschwindigkeit gewonnen worden sind, und derjenigen von Herrn DRUCKer besteht nicht. In Übereinstimmung mit uns und ebenso mit NoYEs und WHITNEY sieht er in ihnen nicht spezifische Konstanten, die die Wechselwirkung zwischen fester Phase und ungesättigter Lösung charakterisieren, sondern Zahlen, die von der Diffusionskonstante des schon gelösten Stoffes abhängen. Mit anderen Worten, in der von ihm hergeleiteten Beziehung (Gleichung 10, Zeitschr. phys. Chem. 36, 696) ist $K$ (Auflösungskonstante) eine Funktion mehrerer Variablen, von denen aber nur eine, $k$ (Diffusionskonstante), von den stofflichen Eigenschaften des Versuchskörpers abhängig ist, andere dagegen sind nur durch die Versuchsanordnung gegeben. Diese von allen denjenigen, die sich mit der Lösungsgeschwindigkeit beschäftigt haben, geteilte Ansicht könnte vielleicht auch eine Stütze in dem Befunde finden, dafs Auflösungsgeschwindigkeitskonstanten so verschiedener Stoffe, wie es die organischen Säuren, Acetanilid und Gips sind, sehr nahe aneinander liegende Zahlen sind. Während die Konstanten chemischer Vorgänge die grölste individuelle Mannigfaltigkeit aufweisen, sind, wie bekannt, die Diffusionskonstanten alle Zahlen derselben Grölsenordnung.

Wenn wir also prinzipiell mit Herrn DRUCKer einverstanden sind, so können wir nicht der von ihm hergeleiteten Beziehung beipflichten, da diese experimentell nícht zugängliche Grö[sen erhält und dort, wo sie sich in einwurfsfreier Weise durch das Experiment prüfen lälst, sich von demselben widerlegt findet. Die Auflösungsgeschwindigkeitskonstante ist vom Volumen der Lösung unabhängig gefunden worden. $\mathrm{Zu}$ seiner Auffassung, dals die Auflösungsgeschwindigkeit umgekehrt proportional dem Volumen zu erfolgen habe, ist Herr Deucker durch die Annahme gekommen, dals an 
der Grenze des festen Stoffes sich eine adhärierende Schicht von der Dicke $s$ bildet. Die Schicht habe unten (unmittelbar am festen Stoffe) die Konzentration $C_{1}$ (die der Sättigung), oben eine Konzentration $C_{2}$, die jedenfalls sehr nahe an $C_{1}^{\prime}$ steht. Der Auflösungsvorgang besteht nach Herm DiUckea: 1. in einer Diffusion längs der Schicht s, 2. in einer Konvelition in der übrigen Lösung. Da die durch $s$ diffundierende Stoffmenge konstant ist, da sie nur durch $s, C_{1}, C_{2}$ bestimmt wird, und diese Menge in verschiedene Volumina durch Konvektion verteilt worden sein kann, so wird daraus natürlich von Herm Drucker gefolgert, dafs die Auflösungsgeschwindigkeit umgekehrt proportional dem Volumen zu erfolgen hat. ${ }^{1}$ Da dies nicht zutrifft, so ist daraus zu schliefsen, dafs die von Herrn Drucker gemachten Annahmen über die Beschaffenheit der adhärierenden Schicht, obgleich im Prinzip ganz wohl möglich, bei den Versuchsbedingungen nicht zutreffen. Wird kräftiges Umdrehen der Wassermenge aufgegeben und der Rührvorrichtung nur die Verteilung des gelösten auf das ganze Volumen zugestanden, wie dies wahrscheinlich bei der früheren für Arsentrioxyd von Herrn Drucker angewandten Methode der Fall war, so kann wohl vorkommen, dafs sich die Annahme des Herrn Drdcker bewähren könnte. Daraus wäre jedoch nur zu schliefsen, dafs die Methode zur Messung der Auflösungsgeschwindigkeit ungeeignet ist. Um die Unabhängigkeit der Auflösungsgeschwindigkeit vom Volumen und die Anwendung der logarithmischen Formel zu rechtfertigen, baben wir übereinstimmend mit Noxess und WHITNEY vorausgesetzt, dấs sich an der lösenden Oberfläche eine äufserst dünne Schicht gesättigter Lösung bildet, aus der der gelöste Stoff für das ganze Volumen geschöpft wird, und die momentan, d. h. in unmelsbar kleiner gegen die für die Konvektion nötige Zeit wieder durch Auflösen des festen Körpers gebildet wird - eine Annahme, die : priori ebenso gerechtfertigt wie die Drucken'sche erscheint. Auch unter dieser Ammahme könnten wir vielleicht einen Einflufs des Volumens auf die Lösungsgeschwindigkeit erwarten, in dem Falle

' Wir wiedergeben den Gedankengang Herm Drocken's, statt sich seiner mathematisclen Ableitung zu bedienen, da dieselbe ziemlieb unklar von Jhm angegeben ist. Es ist uns z. B. unbegreiflich, wie durch irgend welche experimentelle Anordnung die Defination der Konzentration hinfällig sein kann (S. $69 b$ versus 8 )! Ebenso wäre in der Gleichung (10), $v$ konstant behalten, so mufiste $k$ (Auflösungskonstante) von der Konzentration abhängig sein, was doch Herr Drucker wohl nicht behauptet. 
nämlich, wo die Umdrehungsgeschwindigkeit so gesteigert würde, dafs sie die Geschwindigkeit der Wiederbildung der gesättigten Schicht überträfe. Es wäre dann eben auch die zugeführte und auf verschiedene Volumina zu verteilende Stoffmenge konstant. Es lälst sich jetzt nicht angeben, ob dies wohl ausführbar wird.

Hält man an der Auffassung fest, dafs die Auflösungsgeschwindigkeit durch die Diffusionsgeschwindigkeit bedingt wird, so erscheint es unerwartet, dafs die Auflösungsgeschwindigkeit des Marienglases viel kleiner als diejenige des Alabasters ist. Man könnte wohl einwenden, dals die quadratisch gemessene Oberfläche des Alabasters zu klein gefunden wird, da sie durch Erosion, was bei dem Marienglase nicht vorkommt, wesentlich vergrölsert wird. Dies wäre jedoch unrichtig, da auch bei einem und demselben Versuche mit Alabaster, wenn die Platten neu hergestellt gewesen sind, die Erosion stark hervortritt, ohne dafs dies ebenso wie bei den Platten von Benzoësäure vom Einflufs auf die Konstante wäre. Es erscheint uns richtiger, diesen Unterschied auf die Verschiedenheit der Adhäsionsverhältnisse an den glatten und mattierten Oberflächen des Gipses zurückzuführen. Vergleichende Untersuchungen über die Unterschiede der Lösungsgeschwindigkeit mit der Struktur des festen Körpers werden auch vielleicht über die bisher nicht in Betracht gezogene Wechselwirkung zwischen fester Phase und gesättigter Schicht eine Auskunft geben. Es erscheint jedoch überflüssig, näher in diese Betrachtungen einzugehen, bevor die mit grölstmöglicher Drehgeschwindigkeit ausgeführten Versuche uns thatsächlichen Inhalt dazu geben werden.

Wir möchten nur noch kurz zwei von Herrn DRUCKER berührte Punkte betonen. Herr Drucker sagt: ${ }^{1}$,Was die Bemerkung von Bruner und Tolloczko anlangt, dafs ihre Konstante $D$ als Diffusionskoëffizient zu gelten habe, weil die Form der Gleichung dieselbe sei, wie die des Diffusionsgesetzes, so beweist sie gar nichts." Herr DRUCKER scheint jedoch vergessen zu wollen, dafs in der analogen Gleichung auch dieselben Gröfsenwerte einzusetzen sind, wie in der Diffusionsgleichung. Ist doch die Gleichung für die Auflösungsgeschwindigkeit identisch mit der, die z. B. BeILsteIs ${ }^{2}$ für seine Anordnung zur Bestimmung von Diffusionskoëffizienten angewendet hat. Wir glauben, dals zwei Vorgänge, die durch

${ }^{1}$ Zeitschr. phys. Chem. 36, 699.

2 Lieb. Ann. 99, 165; zitiert nach Ostwald, Lehrbuch I, S. 677. 
identische Gleichungen mit denselben zahlenmälsig ausgedrückten GröIsenwerten geregelt werden, für identisch erklärt werden können.

Herr Drucker hebt hervor, dals die Auflösungsgeschwindigkeitskonstante katalytischen Einflüssen unterliege. ${ }^{1}$ Die Untersuchung über die Auflösungsgeschwindigkeit des $\mathrm{As}_{2} \mathrm{O}_{3}$, durch die er diesen Satz als experimentell nachgewiesen zu glauben scheint, ist doch wenig geeignet, den allgemeinen Schlul's absolut gerechtfertigt zu machen. Es ist doch eine chemische Wechselwirkung des $\mathrm{As}_{2} \mathrm{O}_{3}$ mit den Katalysatoren (besonders mit den Basen) keineswegs trotz der unvermehrten Löslichkeit des $\mathrm{As}_{2} \mathrm{O}_{3}$ in den katalysierten Flüssigkeiten - ausgeschlossen. Die Ausnahmestellung dieses Falles hat doch Herr Dnucker selbst nachgewiesen, indem er gefunden hat, dals die Auflösungsgeschwindigkeit des $\mathrm{As}_{2} \mathrm{O}_{33}$ im Gegensatze zu allen übrigen antersuchten Stoffen dem logarithmischen Gesetze nicht unterliegt. Bei $\mathrm{CaSO}_{4}$ und bei Benzoësäure (in einem vorläufigen Versuche, der wegen Unfalles unterbrochen wurde und nicht mitgeteilt worden ist) haben wir keinen Einflufs der H-Ionen, des gewöhnlichsten aller Katalysatoren, finden können. Auch ist es kaum wahrscheinlich, dafs bei der Häufigkeit des Auflösungsvorganges katalytische Einflüsse, falls solche wirklich vorhanden, von der Laboratorium- oder Technikpraxis noch nicht gefunden worden wären. Unseres Wissens nach sind solche noch niemals beobachtet worden.

Die Ergebnisse dieser Untersuchung lassen sich zusammenfassen:

1. Die Auflösungsgeschwindigkeitskonstante ist vom Volum der Lösung unabhängig.

2. Die Rührgeschwindigkeit des Wassers übt einen entscheidenden Einflufs auf die Lösungsgeschwindigkeit.

3. Die Auflösungsgeschwindigkeitskonstante ist auch von der Struktur des sich lösenden Stoffes abhängig.

${ }^{1}$ 1. e. S. 700 .

Krakau, II. chem. Lab. d. Jagiell. Univ., Ende Juli 1901.

Bei der Redaktion eingegangen am 30. August 1901. 